\title{
Biosystems:
}

\section{model synthesis and analysis}

\section{Alex Mazanov}

A mathematical model that incorporates fluctuating time delays and coefficients is also a broad mathematical description of animal production and ecological processes on a "whole system" basis. This model is a first order difference-differential equation which is stated in the most general. terms. However, if a biological process demands that a nonlinear parameter must be used to describe that process which also has a time delay associated with it, solutions to the resulting model may become unstable or periodic as time passes, particularly if the delay is too long, or if the model is too complex.

The homeostasis of any normal, feeding animal or natural ecosystem, together with the extreme complexity of the biochemical processes involved, coupled with known, long, time delays due to, for example, the rate of passage of digesta in sheep, demanded that conditions for mathematical stability of the general model be investigated in a biological context. It was consequently proved that linear, multi-compartmental models of biological systems are mathematically stable independently of the duration of any time delays in the movement of material or energy from one pool to another. This analysis of stability was also proved to be independent of the complexity of the model (that is, independent of the number of pools or the number of possible links between pools) of the biological system. In this model the coefficients and delays may vary as functions of time and "environmental" forces but cannot be functions of pool sizes (since such coefficients would make the model nonlinear). Linear models can, nevertheless, simulate apparently nonlinear responses of biological systems.

Received 15 December 1975. Thesis submitted to the University of New England, October 1974. Degree approved, November 1975. Supervisor: Professor R.A. Leng. 
Two separate numerical methods were developed and implemented for generating numerical solutions to the above mathematical model. The first, effectively a rectangular integration of the equations, treated variable lags. The second was developed for constant time delays in differential equations which were to be solved by means of an available fourth order, variable step, Runge-Kutta numerical integration routine. These routines were used successfully in computer simulations of a range of biological systems. Also, a general line-printer, graph-plotting routine was developed for the visual display of model results together with biological data.

The physical conditions of the conservation of matter and energy, together with the chemical law of mass action were imposed on the biological systems model as constraints on this general, linear, lagdifferential model of biological systems, thus ensuring continued stability with increasing complexity, independently of any time delays. Therefore, stability is shown to be a natural consequence of complexity in biology. 